\title{
Effects of isoflavones on lipid and apolipoprotein levels in patients with type 2 diabetes in Heilongjiang Province in China
}

\author{
Xiao-Xing Chi, ${ }^{1, *}$ Tao Zhang, ${ }^{2}$ Dong-Jie Zhang, ${ }^{1}$ Wei Yu, ${ }^{3}$ Qiu-Ying Wang ${ }^{4}$ and Jing-Long Zhen ${ }^{1}$ \\ ${ }^{1}$ Department of Food College, School of Heilongjiang Bayi Agricultural University, Daqing, Heilongjiang Province 163319, China \\ 2Department of Inspection, Harbin Medical University-Daqing, Daqing, Heilongjiang Province, China \\ ${ }^{3}$ National Coarse Cereals Engineering Research Center, Daqing, Heilongjiang Province, China \\ ${ }^{4}$ Daqing Hospital of Traditional Chinese Medicine, Daqing, Heilongjiang Province, China
}

(Received 30 November, 2015; Accepted 1 February, 2016; Published online 10 June, 2016)

In this study, we examined the association between soy isoflavones and lipid profiles, apolipoprotein levels in patients with type 2 diabetes in China. The study population was composed of 120 cases ( 80 women with type 2 diabetes and 40 healthy women). Objects in treatment group received isoflavones $435 \mathrm{mg} / \mathrm{day}$ for 2 months, then lipid profiles were analyzed by the colorimetry method and apolipoprotein levels were determined by immune turbidimetric method. And all the indexes were determined after oral glucose tolerance test. The levels of total cholesterol, triglyceride and LDL-C significantly reduced and the levels of HDL-C and apolipoprotein A1 significantly raised in the treatment group after intervention $(p<0.05)$. After oral glucose tolerance test, the level of total cholesterol was lower at postprandial $6 \mathrm{~h}$ than at empty stomach in treatment group, it had significantly difference $(p<0.05)$. LDL-C levels in the treatment group not only decreased after intervention, but also was significantly lower at postprandial 4, $6 \mathrm{~h}$ than in non-intervention group. The ratio of apolipoprotein A1/apolipoprotein $B$ at postprandial $2 \mathrm{~h}$ was the highest after treatment in isoflavone group. Supplementation with $435 \mathrm{mg} /$ day of isoflavones exerted favorable effect on the blood total cholesterol, LDL-C levels and the ratio of apolipoprotein A1/apolipoprotein B in Chinese type 2 diabetes women.

Key Words: isoflavones, lipid profiles, apolipoprotein, type 2 diabetes, China

$\mathrm{T}$ ype 2 diabetes has become a global public health challenge. In Southeast Asia, the prevalence has increased three- to fivefold over 30 years and is projected to rise higher. ${ }^{(1,2)}$ Diabetic dyslipidaemia is a cluster of lipid and apolipoprotein abnormalities, which include elevated triacylglycerol-rich lipoproteins (TRLs), an excess of small dense low density lipoprotein-cholesterol (LDL) particles (independent of the concentration of LDL-cholesterol) and reduced concentrations of both high density cholesterol (HDL-C) and the major HDL apolipoprotein, apolipoprotein A1 (ApoA1). ${ }^{(3,4)}$ Reduction of LDL-C is the first target of lipidaltering therapy in diabetes, secondly is the elevated typical dyslipidemia triglycerides and reduced HDL-C. This is mainly because some studies often find LDL-C to be the strongest predictor of coronary heart disease risk, not only because of a larger evidence base for LDL-C lowering. ${ }^{(5,6)}$ Lifestyle change, nutrition therapy, and improved glycemic control have some impact on lipids in patients with diabetes and in metabolic syndrome, the levels of LDL-C in most of them reduce 0.6 to $0.9 \mathrm{mmol} / \mathrm{L}^{(7)}$ and levels of triglycerides reduce $0.2 \mathrm{mmol} / \mathrm{L}$ or less. ${ }^{(8,9)}$

As TRL and LDL particles contain a single molecule of apolipoprotein $\mathrm{B}(\mathrm{ApoB})$, the concentration of $\mathrm{ApoB}$ in plasma may be a better indicator of cardiovascular risk than the simple measurement of LDL-cholesterol. ${ }^{(10-13)}$ The ApoB:ApoA1 ratio was better than traditional lipid and apolipoprotein levels as a predictor of CVD in large case-control studies ${ }^{(14,15)}$ and in people treated with statins. ${ }^{(16)}$ These analyses indicate that the standard lipids should be replaced by assessment of ApoB and ApoA1, particularly in people with diabetes. ${ }^{(17-19)}$ The search case-control study showed that ApoB levels were elevated in type 2 diabetes compared to nondiabetic controls independent of LDL-C. ${ }^{(20,21)}$ For that reason, measurement of ApoB in addition to LDL-C may help reclassify CVD risk in type 2 diabetes patients with borderline LDL-C levels and also determine when lipid-lowering treatment should be considered.

Isoflavones are phytoestrogens present abundantly in soybean, associated with various beneficial health properties such as antiestrogenic, reducing hormone dependent cancers and bone resorption. It shows healing properties in relation to cardiovascular diseases, renal diseases, osteoporosis and diabetes. However, because the doses of isoflavones from soy products used in animal or in vitro studies are much higher than those in the soy products regularly consumed by humans, the health benefits of soy for type 2 diabetes have been found in epidemiological studies to be limited and inconsistent. ${ }^{(22-25)}$ Whether the effects of isoflavones on lipid profiles and apolipoprotein levels differ between nonintervention and isoflavones treatment on patients with type 2 diabetes remains to be determined. The aim of the present study was to investigate two hypotheses: one is that isoflavones have observable effects on lipid profiles and apolipoprotein levels, such as TG, TC, LDL-C, HDL-C and ApoA1, ApoB, the other one is that these effects are modified by different oral glucose tolerance in the patients with type 2 diabetes of Chinese population.

\section{Materials}

Subjects. In a double, blind, randomized trial, eighty patients with type 2 diabetes who met with the research criteria were divided into diabetes mellitus non-intervention group (DM-NIG) and isoflavones dose group (Iso-G). As the same time, forty normal subjects joined this protocol and were taken as control group (CG). These patients came from Daqing Chinese medicine hospital and the first affiliated hospital of Heilongjiang Chinese Medicine University of Heilongjiang province in China. The women who received hormone replacement therapy (androgen, estrogen, progesterone, and respectively related synthetic drugs)

*To whom correspondence should be addressed. E-mail: chixiaoxing@sina.com 
or who took medication (selective serotonin reuptake inhibitors, such as escitalopram oxalate, fluoxetine hydrochloride, fluvoxamine maleate, paroxetine hydro-chloride, paroxetine and sertraline hydrochloride) or health functional foods containing isoflavones or sophoricosides, known to have influence on estrogen levels were excluded. The subjects who had a history of cancer within 5 years, liver and kidney diseases or thyroid disorder, or who had an allergy to soybeans were also excluded. Furthermore, subjects cured with cholesterol lowering drugs within 2 months were excluded. All subjects provided written informed consent for participation in the study. The research protocol was approved by the Ethical Committee of the Heilongjiang Center for Disease Control and Prevention.

It is important to point out the presence of about $145 \mathrm{mg}$ of soy isoflavones in each capsule (aglycone equivalents) of which genistein and daidzein comprised $52.2 \%$ and $47.8 \%$, respectively, as evidenced by high performance liquid chromatography (HPLC) analysis, addition to $5 \mu \mathrm{g}$ of Vitamin D, providing $100 \%$ of the daily recommended dose. The placebo capsules were identical in size and color, contained starch and Vitamin from the active formulation. Isoflavones and placebo supplied by Heilongjiang Datong Company and supervised by Heilongjiang Society for Nutrition. The treatment code was allocated randomly. The randomization number with treatment assignment for the individual subject was blinded for the duration of the trial. The subjects received either three isoflavones or three placebo capsules daily for 2 months. The total dose of isoflavone was thus about $435 \mathrm{mg} /$ day. Isoflavones dose in this study was either more or less compared with previous studies. We chose the dose was due to that we found isoflavones at the dose of $435 \mathrm{mg}$ /day could significantly improve the levels of some antioxidant indicators, such as SOD, GSH-PX and GSH-ST of hyperlipidemia patients in our previous research. At the same time, some scholars had found that antioxidants could reduce the oxidation of lipid and glucose, ${ }^{(26,27)}$ so we guessed whether soy isoflavones at the current dose had effect on the blood lipid index in patients with type 2 diabetes. During the supplemental period, isoflavones intake from foods was restricted to less than $10 \mathrm{mg} /$ day. The subjects received education on food sources of isoflavones by a dietician.

General characteristics and anthropometric measures. The information on the general characteristics of the subjects was collected through face to face interviews. The anthropometric data were gathered using standard methods. The body weight $(\mathrm{kg})$ and height $(\mathrm{cm})$ were measured to the nearest $0.1 \mathrm{~kg}$ and $0.1 \mathrm{~cm}$, respectively. The body mass index (BMI) was calculated by dividing the body weight $(\mathrm{kg})$ by the squared height $\left(\mathrm{m}^{2}\right)$. The waist and hip circumferences were measured twice with a flexible steel metric tape to the nearest $0.1 \mathrm{~cm}$.

Analysis of blood parameters. Blood was collected in the morning from a mid-arm vein after overnight fasting $(12 \mathrm{~h})$ before and after protocol. Then oral glucose tolerance test carried out, after $12 \mathrm{~h}$ of overnight fasting, all participants underwent a $6 \mathrm{~h}$ $75 \mathrm{~g}$ OGTT. After the blood was centrifuged at $3,000 \times g$ at $4{ }^{\circ} \mathrm{C}$ for $10 \mathrm{~min}$, the supernatant was stored at $-80^{\circ} \mathrm{C}$ until it was assayed. The blood glucose levels were analyzed using the enzymatic method (Bayer, Tokyo, Japan). The serum TC, TG, LDL-C and HDL-C levels were analyzed using the colorimetry method (Bayer, Berkeley, CA). The Apo A1 and Apo B levels were analyzed by immune turbidimetric method (PACKARD, Emeryville, CA).

Statistical analysis. Statistical analysis was performed using statistical analysis software (SAS) ver. 9.1.3 (SAS Institute INC., Cary, NC). The categorical variables between the two groups were compared with the chi-square test and the data were presented as a percent or number. Also, continuous variables between two groups or within a group were compared with independent or paired $t$ test, respectively, and data were presented as mean \pm SD. Statistical significance was defined at $p<0.05$.

\section{Results}

The general characteristics and anthropometric measures of the subjects are shown in Table 1. The mean ages, the height, the weights, body mass index, the waist circumference, the hip circumference, waist to hip ratio, levels of fasting blood glucose and levels of glycosylated serum protein of subjects were not significantly different within the three groups.

Table 2 shows the levels of lipid profiles and apolipoprotein before and after intervention. Before intervention, the levels of lipid profiles and apolipoprotein in DM-NIG and Iso-G had no significant difference with CG $(p>0.05)$. On the other hand, after intervention, the levels of TC, TG, LDL-C, HDL-C, ApoAl and ApoB were not changed in $\mathrm{CG}$ and in Iso-G. Isoflavones decreased the levels of TC, TG and LDL-C, while increased the levels of HDL-C and ApoAl significantly in the treatment group after intervention $(p<0.05)$. The level of ApoB also rose in treatment group after intervention, but the difference was nonsignificant.

The changes of lipid profiles levels after OGTT are shown in Fig. 1. The levels of TG were higher in DM-NIG and Iso-G than in CG before and after OGTT. In DM-NIG, TG levels were higher

Table 1. Baseline characteristics of the participants with type 2 diabetes and the controls (mean \pm SD)

\begin{tabular}{|c|c|c|c|c|c|c|c|c|c|}
\hline Groups & $\begin{array}{c}\text { Age } \\
\text { (years old) }\end{array}$ & $\begin{array}{l}\text { Height } \\
(\mathrm{cm})\end{array}$ & $\begin{array}{l}\text { Weight } \\
(\mathrm{kg})\end{array}$ & $\underset{\left(\mathrm{kg} / \mathrm{m}^{2}\right)}{\text { Body mass index }}$ & $\begin{array}{c}\text { Waist } \\
\text { circumference } \\
(\mathrm{cm})\end{array}$ & $\begin{array}{l}\text { Hip circumference } \\
(\mathrm{cm})\end{array}$ & $\begin{array}{l}\text { Waist to } \\
\text { hip ratio }\end{array}$ & $\begin{array}{l}\text { Fasting blood } \\
\text { glucose level } \\
\text { (mmol/L) }\end{array}$ & $\begin{array}{l}\text { Glycosylated } \\
\text { serum protein } \\
\text { level }(\mu \mathrm{g} / \mathrm{L})\end{array}$ \\
\hline CG & $46.5 \pm 13.8$ & $162.36 \pm 2.81$ & $62.52 \pm 3.71$ & $23.70 \pm 0.89$ & $89.45 \pm 3.13$ & $95.37 \pm 3.09$ & $0.94 \pm 0.05$ & $5.01 \pm 0.36$ & $162.3 \pm 28.36$ \\
\hline DM-NIG & $48.5 \pm 13.1$ & $164.58 \pm 3.71$ & $62.49 \pm 2.84$ & $23.07 \pm 0.72$ & $87.95 \pm 2.04$ & $95.98 \pm 2.11$ & $0.92 \pm 0.03$ & $10.33 \pm 2.32$ & $234.5 \pm 65.32$ \\
\hline Iso-G & $54.87 \pm 8.97$ & $161.08 \pm 3.03$ & $63.38 \pm 2.48$ & $24.43 \pm 0.87$ & $90.45 \pm 3.18$ & $97.81 \pm 2.10$ & $0.92 \pm 0.02$ & $9.86 \pm 4.53$ & $238.64 \pm 57.25$ \\
\hline
\end{tabular}

Experimental conditions and treatment procedures are given in Materials and Methods. Graph depicts (mean \pm SD).

Table 2. Levels of lipid profiles and apolipoprotein levels before and after intervention (mean \pm SD)

\begin{tabular}{|c|c|c|c|c|c|c|c|}
\hline Groups & & $\mathrm{TC}(\mathrm{mmol} / \mathrm{L})$ & TG (mmol/L) & LDL-C (mmol/L) & $\mathrm{HDL}-\mathrm{C}(\mathrm{mmol} / \mathrm{L})$ & ApoA1 (g/L) & ApoB (g/L) \\
\hline \multirow[t]{2}{*}{ CG } & Before & $4.71 \pm 1.21$ & $1.77 \pm 1.17$ & $2.68 \pm 0.74$ & $1.45 \pm 0.42$ & $1.15 \pm 0.18$ & $0.58 \pm 0.14$ \\
\hline & After & $4.69 \pm 1.04$ & $1.71 \pm 0.7$ & $2.69 \pm 0.57$ & $1.59 \pm 0.26$ & $1.15 \pm 0.16$ & $0.61 \pm 0.15$ \\
\hline \multirow[t]{2}{*}{ DM-NIG } & Before & $4.98 \pm 0.86$ & $1.87 \pm 1.02$ & $2.63 \pm 0.38$ & $1.52 \pm 0.53$ & $1.12 \pm 0.19$ & $0.58 \pm 0.17$ \\
\hline & After & $4.78 \pm 0.98$ & $1.56 \pm 0.88$ & $2.57 \pm 0.43$ & $1.53 \pm 0.23$ & $1.15 \pm 0.14$ & $0.59 \pm 0.16$ \\
\hline \multirow[t]{2}{*}{ Iso-G } & Before & $5.12 \pm 1.21$ & $1.97 \pm 1.17$ & $2.78 \pm 0.74$ & $1.35 \pm 0.42$ & $1.05 \pm 0.18$ & $0.54 \pm 0.14$ \\
\hline & After & $4.79 \pm 1.04 *$ & $1.49 \pm 0.7$ * & $2.39 \pm 0.57$ * & $1.52 \pm 0.56$ * & $1.18 \pm 0.18 *$ & $0.63 \pm 0.15$ \\
\hline
\end{tabular}

Experimental conditions and treatment procedures are given in Materials and Methods. Graph depicts (mean \pm SD). Asterisk means significant against before intervention $\left({ }^{*} p<0.05\right), t$ test. 

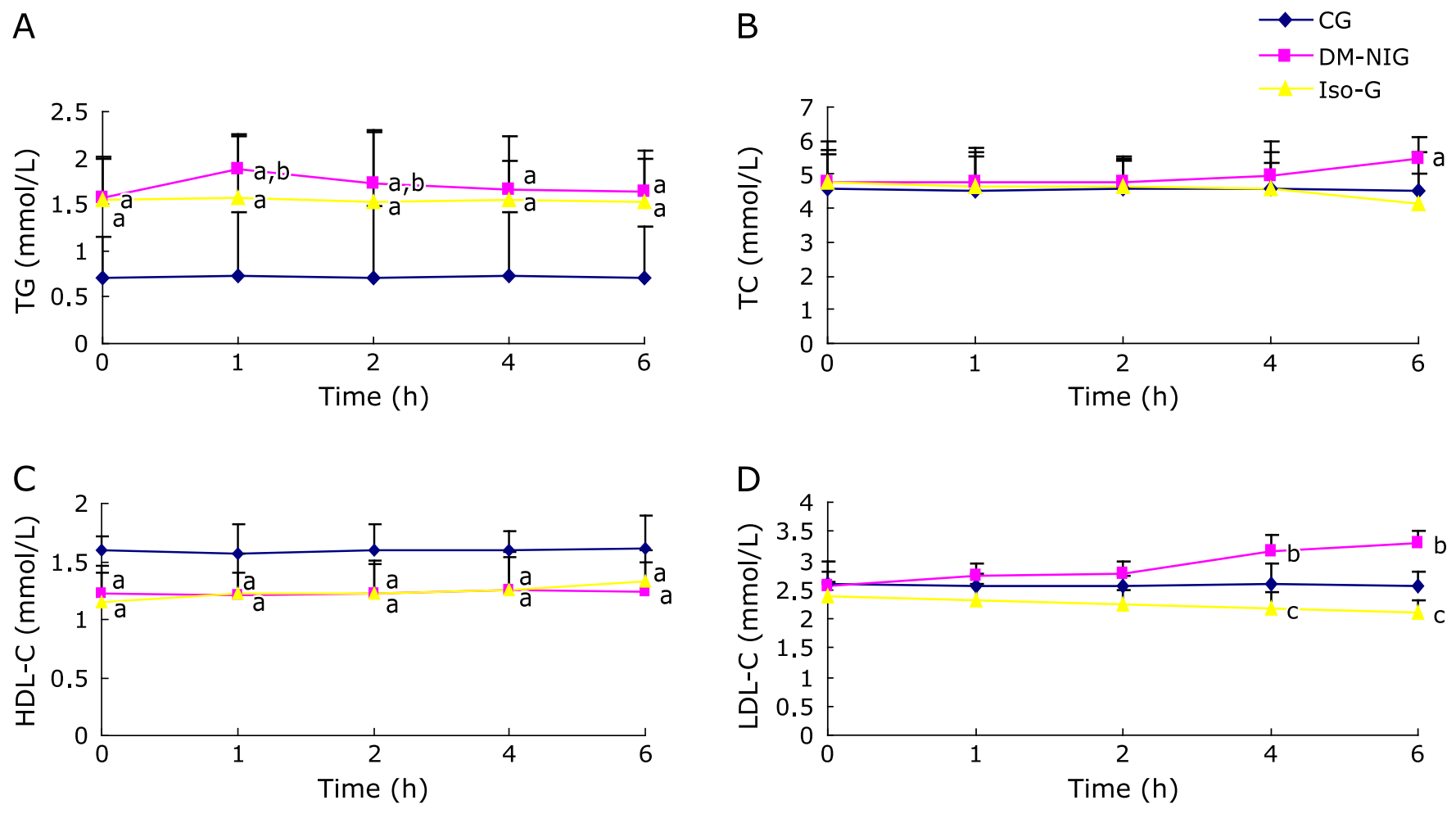

Fig. 1. Levels of TG (A), TC (B), HDL-C (C) and LDL-C (D) in each group after OGTT are shown. Experimental conditions and treatment procedures are given in Materials and Methods. Data are expressed as mean \pm SD. ${ }^{a} p<0.05$ compared with $C G,{ }^{b} p<0.05$ compared with empty stomach, ${ }^{\mathrm{C}} p<0.05$ compared with DM-NIG.

at postprandial $1,2 \mathrm{~h}$ than at empty stomach, but it had no obvious changes in Iso-G after OGTT. The level of TC was higher in DM-NIG than in CG at postprandial $6 \mathrm{~h}$. Significantly, it showed that the level of TC was lower at postprandial $6 \mathrm{~h}$ than at empty stomach in Iso-G, it had significantly difference $(p<0.05)$. There were no changes of HDL-C levels after OGTT in each group. The level of LDL-C was higher at postprandial $4,6 \mathrm{~h}$ than at empty stomach in DM-NIG, the change was significantly different $(p<0.05)$. It was worth mentioning that LDL-C levels in the treatment group were not only decreased after intervention, but also were significantly lower at postprandial $4,6 \mathrm{~h}$ than in DM-NIG.

Levels of ApoA1 in each group after OGTT (mean \pm SD) are shown in Fig. 2. In control group, the level of ApoA1 at empty stomach, postprandial $1,2,4$ and $6 \mathrm{~h}$ was $1.13 \pm 0.12,0.13 \pm 1.18$, $1.11 \pm 0.19,1.10 \pm 0.15$ and $1.12 \pm 0.17 \mathrm{~g} / \mathrm{L}$, respectively. In diabetes mellitus non-intervention group, the levels of ApoA1 was $1.04 \pm 1.15,1.07 \pm 0.15,1.07 \pm 0.14,1.13 \pm 0.19$ and $1.05 \pm$ $0.16 \mathrm{~g} / \mathrm{L}$, respectively. In isoflavones dose group, the level of ApoA1 was $1.13 \pm 0.18,1.18 \pm 0.19,1.16 \pm 0.11,1.11 \pm 0.16$ and $1.12 \pm 0.15 \mathrm{~g} / \mathrm{L}$, respectively. There was no significantly different of ApoA1 level after OGTT in every group.

Levels of ApoB in each group after OGTT (mean \pm SD) is shown in Fig. 3. In control group, the levels of ApoB at empty stomach, postprandial $1,2,4$ and $6 \mathrm{~h}$ was $0.65 \pm 0.12,0.64 \pm 1.12$, $0.62 \pm 0.17,0.62 \pm 0.12$ and $0.60 \pm 0.12 \mathrm{~g} / \mathrm{L}$, respectively. In diabetes mellitus non-intervention group, the level of ApoB was $0.68 \pm 1.15,0.69 \pm 0.14,0.75 \pm 0.18,0.80 \pm 0.18$ and $0.88 \pm 0.19$ $\mathrm{g} / \mathrm{L}$, respectively. As can be seen from the results, the level of ApoB in DM-NIG was the highest of all the groups at postprandial $6 \mathrm{~h}$ and compared with empty stomach, the different was significantly $(p<0.05)$. In isoflavones dose group, the level of ApoB was $0.69 \pm 0.18,0.71 \pm 0.15,0.71 \pm 0.13,0.70 \pm 0.18$ and $0.65 \pm 0.16$ $\mathrm{g} / \mathrm{L}$, respectively. Compared with empty stomach, the level of ApoB at postprandial $6 \mathrm{~h}$ rose significantly $(p<0.05)$.

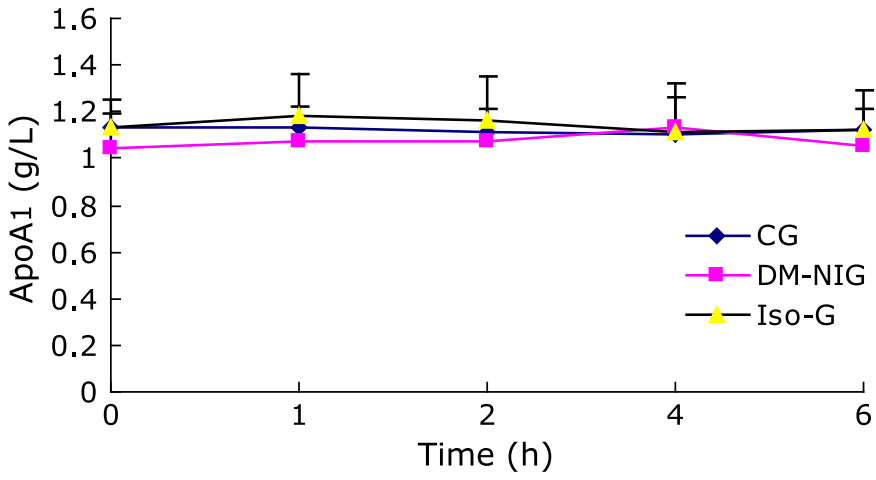

Fig. 2. Levels of ApoA1 in each group after OGTT are shown. Experimental conditions and treatment procedures are given in Materials and Methods. Data are expressed as mean \pm SD. ${ }^{a} p<0.05$ compared with CG, ${ }^{\mathrm{b}} p<0.05$ compared with empty stomach, ${ }^{c} p<0.05$ compared with DM-NIG.

Ratio of ApoA1/ApoB in each group after OGTT is shown in Fig. 4. In control group, ratio of $\mathrm{Apo} A 1 / \mathrm{ApoB}$ at empty stomach, postprandial $1,2,4$ and $6 \mathrm{~h}$ was $1.85 \pm 0.47,1.87 \pm 0.45,1.87 \pm$ $0.54,1.86 \pm 0.55$ and $1.82 \pm 0.45$, respectively. In diabetes mellitus non-intervention group, ratio of ApoA1/ApoB was $1.64 \pm 0.40$, $1.63 \pm 0.45,1.63 \pm 0.44,1.65 \pm 0.49$ and $1.45 \pm 0.46$, respectively. In isoflavones dose group, ratio of $\mathrm{Apo} A 1 / \mathrm{ApoB}$ was $1.63 \pm 0.48,1.73 \pm 0.39,1.86 \pm 0.31,1.61 \pm 0.46$ and $1.58 \pm 0.45$, respectively. It showed that after OGTT, obvious changes of the ratio of $A$ poA $1 / A p o B$ had taken place. We can see that the ratio of ApoA1/ApoB was lower at postprandial $6 \mathrm{~h}$ than at empty stomach in DM-NIG. Obviously, the ratio of ApoA1/ApoB at postprandial $2 \mathrm{~h}$ was the highest $(1.86 \pm 0.31)$ after treatment in Iso-G. 


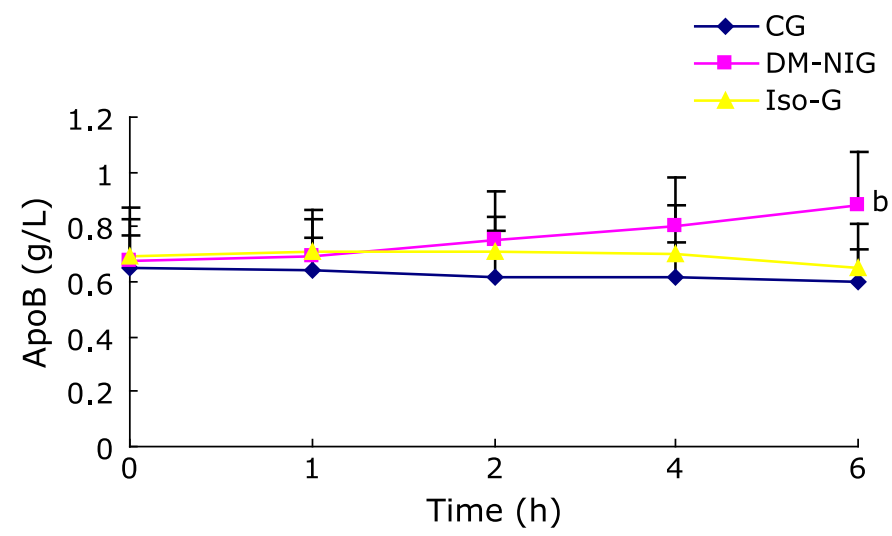

Fig. 3. Levels of $A p o B$ in each group after OGTT are shown. Experimental conditions and treatment procedures are given in Materials and Methods. Data are expressed as mean \pm SD. ${ }^{a} p<0.05$ compared with CG, ${ }^{b} p<0.05$ compared with empty stomach, ${ }^{c} p<0.05$ compared with DM-NIG.

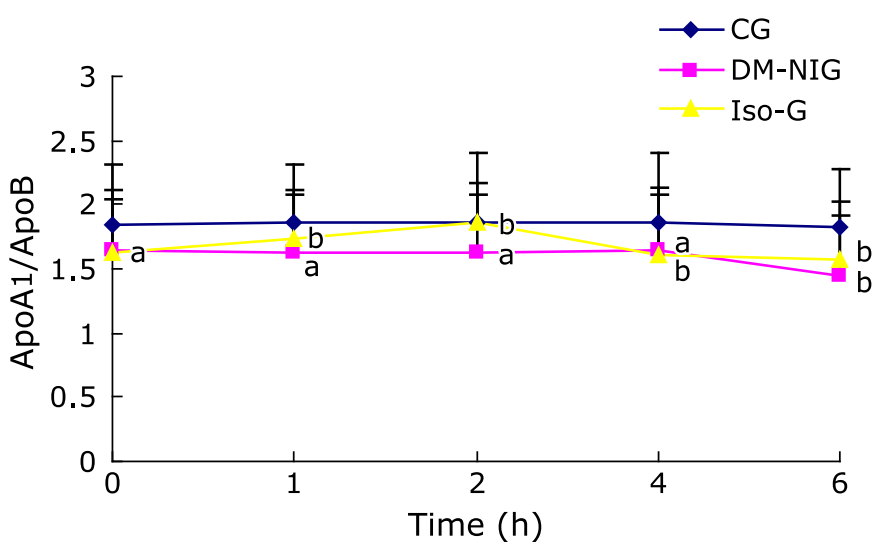

Fig. 4. Ratio of $A p o A 1 / A p o B$ in each group after OGTT are shown. Experimental conditions and treatment procedures are given in Materials and Methods. Data are expressed as mean \pm SD. ${ }^{a} p<0.05$ compared with $C G,{ }^{b} p<0.05$ compared with empty stomach, ${ }^{c} p<0.05$ compared with DM-NIG.

\section{Discussion}

This study explored the effects of supplementation of isoflavones on the lipid profiles and apolipoprotein levels related to type 2 diabetes mellitus in the Chinese women. We found that oral supplement of isoflavones at a dose of $435 \mathrm{mg} /$ day for 2 months could decrease the levels of TC, TG, and LDL-C, while increased HDL-C and ApoA1 levels in serum. In addition to improving lipid profiles in patients with diabetes mellitus, this analysis found that, compared with placebo, treatment with isoflavones also significantly improved TC, LDL-C levels and the ratio of ApoA1/ ApoB after OGTT.

In October 1999, a health claim put forward by the US food and drug administration (FDA) was based on the results of a clinical trial suggesting that at least $25 \mathrm{~g}$ of soy protein per day reduced total-C and LDL-C and it approved that products containing soy protein could protective against CHD. Also, the AHA Nutrition committee reevaluated the evidence on isoflavone and CVD and updated its science advisory in 2006. They reported that only 3 studies (among 19 studies) of soy isoflavones showed a reduction in LDL-C; however, no significant changes in HDL-C and TG were observed. ${ }^{(28)}$ Although the evidence for improving lipid profiles and apolipoprotein levels effect of isoflavones on patients with 2 type diabetes is clear from animal or in vitro studies, the results from epidemiological studies have been inconsistent, and we need to consider these inconsistencies further. The primary defect underlying abnormalities in lipoprotein transport in type 2 diabetes is insulin resistance, which leads to increased triglycerides in the fasting and postprandial states, preponderance of small, dense LDL and low concentrations of dysfunctional HDL. Concentrations of LDL-C are most often not remarkably abnormal. Based on this lipoprotein profile, it seems somewhat counterintuitive to prioritize LDL-C lowering in type 2 diabetes rather than simply cholesterol content. Also, supplementation with a soy protein containing isoflavones significantly reduced levels of TC, LDL-C and TG and increased levels of HDL-C. ${ }^{(29)}$ In our study, isoflavones supplements ( $435 \mathrm{mg} /$ day) significantly reduced the levels of TC, TG, LDL-C, while raised the levels of HDL-C two months later. And after OGTT, the levels of TC and LDL-C significantly reduced at postprandial $6 \mathrm{~h}$ than at empty stomach, which suggested that isoflavones could improve the lipid profiles of patients with 2 type diabetes. However, other lipid profiles and levels were not affected by the supplementation.

Several studies have indicated that ApoAl and ApoB provide better discrimination of CHD risk than serum LDLC, HDLC and non-HDLC in cardiovascular disease and CHD prevention. ${ }^{(30)} \mathrm{A}$ randomised primary prevention trial of lovastatin also get a similar conclusion in a non-diabetic population. ${ }^{(31)} \mathrm{ApoB}$ is essential for the binding of LDL-C particles, allowing cells to internalize LDL-C and thus absorb cholesterol. An excess of ApoBcontaining particles highly correlates with the level of non-HDL-C cholesterol and it is a main trigger in the atherogenic process. ${ }^{(32)}$ On the other hand, levels of ApoA1 strongly correlate with those of HDL-C, and expression of ApoA1 may be largely responsible for determining the plasma level of HDL-C, protecting against CVD. Notably, the calculated ApoB/ApoA1 ratio has been consistently reported as a prognostic cardiovascular risk factor superior than LDL-C. Willcox founded that the ratio of ApoA1 and ApoB should on behalf of the comprehensive changes of the two lipoprotein ApoAl and ApoB, which could be considered to be an important dangerous forecast factors of Diabetic coronary heart disease. ${ }^{(33)}$ And the rising of the ratio of $A p o A 1$ and $A p o B$ is beneficial to type 2 diabetes. Some scholars found that the peak post-load glucose (PPG) was about $1 \mathrm{~h}$ in healthy individuals. But in type 2 diabetes patients, blood glucose continues rose slowly, which due to insulin secretion defect and insulin resistance, so PPG reached a peak delayed to $1.5 \sim 2 h{ }^{(34)}$ Lind found that the ratio of ApoA1/ApoB had positive correlation with insulin sensitivity. ${ }^{(35)}$ Isoflavones could enhance insulin sensitivity, so the ratio of ApoA1/ApoB was highest at postprandial $2 \mathrm{~h}$ in Iso-G. To our knowledge, it is for the first time that our study demonstrated the favorable impact of isoflavones on ApoB levels and ApoA1/ ApoB ratio in the diabetic population. We found that the ratio of ApoA $1 / A p o B$ is the highest at postprandial $2 \mathrm{~h}$ after treatment with isoflavone. In general, blood glucose levels reached the highest peak at 1.5 to $2 \mathrm{~h}$ after OGTT. Some cell culture experiments found that insulin sensitivity increased at $1 \sim 3 \mathrm{~h}$ after OGTT, and the adhesion of insulin and fat cells became stronger. Lind found that the ratio of $\mathrm{ApoA} 1 / \mathrm{ApoB}$ had positive correlation with insulin sensitivity. Isoflavones could enhance insulin sensitivity, so the ratio of $A p o A 1 / A p o B$ was highest at postprandial $2 \mathrm{~h}$ in Iso-G. These findings were consistent with our results. On the contrary, it was the lowest at postprandial $6 \mathrm{~h}$ without treatment. Although the influence of isoflavones on ApoAl and ApoB was non-significant, but it made the ratio of ApoA1 and ApoB changed.

In conclusion, supplementation with $435 \mathrm{mg} /$ day of isoflavones exerted favorable effect on the blood TC, LDL-C levels and the ratio of $\mathrm{ApoA} 1 / \mathrm{ApoB}$ in Chinese women with type 2 diabetes. Further studies are needed to evaluate the clinical implication of these positive effects in women with abnormal blood glucose. 


\section{Acknowledgments}

This research was supported by funding from the National Natural Science Foundation of China (No. 81102136) and agricultural products processing and quality safety innovation universities team in heilongjiang province (2014TD006). We thank Dr. Cui and Dr. Zhang for their assistance with directing the study. Xiao-Xing Chi and Tao Zhang have contributed equally to this work.

\section{Abbreviations}

ApoA1 apolipoprotein A1

ApoB apolipoprotein B

BMI body mass index

\section{References}

1 Shaw JE, Sicree RA, Zimmet PZ. Global estimates of the prevalence of diabetes for 2010 and 2030. Diabetes Res Clin Pract 2010; 87: 4-14.

2 Yoon KH, Lee JH, Kim JW, et al. Epidemic obesity and type 2 diabetes in Asia. Lancet 2006; 368: 1681-1688.

3 Taskinen MR. Type 2 diabetes as a lipid disorder. Curr Mol Med 2005; 5: 297-308.

4 Mooradian AD. Dyslipidemia in type 2 diabetes mellitus. Nat Clin Pract Endocrinol Metab 2009; 5: 150-159.

5 Turner RC, Millns H, Neil HA, et al. Risk factors for coronary artery disease in non-insulin dependent diabetes mellitus: United Kingdom Prospective Diabetes Study (UKPDS: 23). BMJ 1998; 316: 823-828.

6 Lehto S, Rönnemaa T, Haffner SM, Pyörälä K, Kallio V, Laakso M. Dyslipidemia and hyperglycemia predict coronary heart disease events in middle-aged patients with NIDDM. Diabetes 1997; 46: 1354-1359.

7 American Diabetes Association. Standards of medical care in diabetes--2006. Diabetes Care 2006; 29 Suppl 1: S4-S42.

8 Orchard TJ, Temprosa M, Goldberg R, et al. The effect of metformin and intensive lifestyle intervention on the metabolic syndrome: the Diabetes Prevention Program randomized trial. Ann Intern Med 2005; 142: 611-619.

9 Lindstrom J, Eriksson JG, Valle TT, et al. Prevention of diabetes mellitus in subjects with impaired glucose tolerance in the Finnish Diabetes Prevention Study: results from a randomized clinical trial. J Am Soc Nephrol 2003; 14 (7 Suppl 2): S108-S113.

10 Sniderman A. Targets for LDL-lowering therapy. Curr Opin Lipidol 2009; 20: $282-287$.

11 Lau JF, Smith DA. Advanced lipoprotein testing: recommendations based on current evidence. Endocrinol Metab Clin North Am 2009; 38: 1-31.

12 Contois JH, McConnell JP, Sethi AA, et al.; AACC Lipoproteins and Vascular Diseases Division Working Group on Best Practices. Apolipoprotein B and cardiovascular disease risk: position statement from the AACC Lipoproteins and Vascular Diseases Division Working Group on Best Practices. Clin Chem 2009; 55: 407-419.

13 Barter PJ, Ballantyne CM, Carmena R, et al. Apo B versus cholesterol in estimating cardiovascular risk and in guiding therapy: report of the thirtyperson/ten-country panel. J Intern Med 2006; 259: 247-258.

14 Walldius G, Jungner I, Holme I, Aastveit AH, Kolar W, Steiner E. High apolipoprotein B, low apolipoprotein A-I, and improvement in the prediction of fatal myocardial infarction (AMORIS study): a prospective study. Lancet 2001; 358: 2026-2033.

15 McQueen MJ, Hawken S, Wang X, et al. Lipids, lipoproteins, and apolipoproteins as risk markers of myocardial infarction in 52 countries (the INTERHEART study): a case-control study. Lancet 2008; 372: 224-233.

16 Holme I, Cater NB, Faergeman O, et al.; Incremental Decrease in End-Points Through Aggressive Lipid-Lowering (IDEAL) Study Group. Lipoprotein predictors of cardiovascular events in statin-treated patients with coronary heart disease. Insights from the Incremental Decrease in End-points Through Aggressive Lipid-lowering Trial (IDEAL). Ann Med 2008; 40: 456-464.

17 Sniderman AD. Low-density lipoprotein lowering in type 2 diabetes mellitus: how to know how low to go. Curr Opin Endocrinol Diabetes Obes 2007; 14: 116-123.

18 Sniderman AD, Faraj M. Apolipoprotein B, apolipoprotein A-I, insulin resis-
CG control group

DM-NIG diabetes mellitus non-intervention group

HDL-C high density lipoprotein cholesteroll

HPLC high performance liquid chromatography

Iso-G isoflavone dose group

LDL low density lipoprotein cholesterol

OGTT oral glucose tolerance test

TC the serum total cholesterol

TG triglyceride

TRLs triacylglycerol-rich lipoproteins

\section{Conflict of Interest}

No potential conflicts of interest were disclosed. tance and the metabolic syndrome. Curr Opin Lipidol 2007; 18: 633-637.

19 Davidson MH. Is LDL-C passed its prime? The emerging role of non-HDL, LDL-P, and ApoB in CHD risk assessment. Arterioscler Thromb Vasc Biol 2008; 28: 1582-1583.

20 Miller M, Ginsberg HN, Schaefer EJ. Relative atherogenicity and predictive value of non-high-density lipoprotein cholesterol for coronary heart disease. Am J Cardiol 2008; 101: 1003-1008.

21 Rosenson RS. Management of non-high-density lipoprotein abnormalities. Atherosclerosis 2009; 207: 328-335.

22 Mueller NT, Odegaard AO, Gross MD, et al. Soy intake and risk of type 2 diabetes mellitus in Chinese Singaporeans: soy intake and risk of type 2 diabetes. Eur J Nutr 2012; 51: 1033-1040.

23 Morimoto Y, Steinbrecher A, Kolonel LN, Maskarinec G. Soy consumption is not protective against diabetes in Hawaii: the Multiethnic Cohort. Eur $J$ Clin Nutr 2011; 65: 279-282.

24 Villegas R, Gao YT, Yang G, et al. Legume and soy food intake and the incidence of type 2 diabetes in the Shanghai Women's Health Study. Am J Clin Nutr 2008; 87: 162-167.

25 Nanri A, Mizoue T, Takahashi Y, et al. Soy product and isoflavone intakes are associated with a lower risk of type 2 diabetes in overweight Japanese women. J Nutr 2010; 140: 580-586.

26 Koo JR, Vaziyi ND. Effects of diabetes, insulin and antioxidants on NO synthase abundance and No interaction with reactive oxygen species. Kidney Int 2003; 63: 195-201.

27 Koo JR, Ni Z, Oviesi F, Vaziri ND. Antioxidant therapy potentiates antihypertensive action of insulin in diabetic rats. Clin Exp Hypertens 2002; 24 333-344.

28 Sacks FM, Lichtenstein A, Van Horn L, Harris W, Kris-Etherton P, Winston M; American Heart Association Nutrition Committee. Soy protein, isoflavones, and cardiovascular health: an American Heart Association Science Advisory for professionals from the Nutrition Committee. Circulation 2006; 113: $1034-1044$.

29 Zhan S, Ho SC. Meta-analysis of the effects of soy protein containing isoflavones on the lipid profile. Am J Clin Nutr 2005; 81: 397-408.

30 Liting P, Guoping L, Zhenyue C. Apolipoprotein B/apolipoprotein A1 ratio and non-high-density lipoprotein cholesterol. Herz 2015; 40 Suppl 1: 1-7.

31 Chariton-Menys V, Betteridge DJ, Colhoun H, et al. Apolipoproteins, cardiovascular risk and statin response in type 2 diabetes: the Collaborative Atorvastatin Diabetes Study (CARDS). Diabetologia 2009; 52: 218-225.

32 Cromwell WC, Barringer TA. Low-density lipoprotein and apolipoprotein B: clinical use in patients with coronary heart disease. Curr Cardiol Rep 2009; 11: $468-475$.

33 Willcox JK, Catignani GL, Lazarus S. Tomatoes and cardiovascular health. Crit Rev Food Sci Nutr 2003; 43: 1-18.

34 Abdul-Ghani MA, Jenkinson CP, Richardson DK, Tripathy D, DeFronzo RA. Insulin secretion and action in subjects with impaired fasting glucose and impaired glucose tolerance: results from the Veterans Administration Genetic Epidemiology Study. Diabetes 2006; 55: 1430-1435.

35 Lind L, Vessby B, Sundström J. The apolipoportein B/AI ratio and the metabolic syndrome independently predict risk for myocardial infarction in middle-age men. Arterioscler Thromb Vasc Biol 2006; 26: 406-410. 